\title{
Luminescence of black silicon
}

\author{
Ali Serpengüzel, ${ }^{1}$ Adnan Kurt, ${ }^{1}$ Ibrahim Inanç, ${ }^{2}$ James E. Cary, ${ }^{3}$ and \\ Eric Mazur ${ }^{4}$ \\ ${ }^{1}$ Koç University, Department of Physics, Microphotonics Research Laboratory, \\ Rumelifeneri Yolu, Sariyer, Istanbul 34450 Turkey \\ ${ }^{2}$ Sabancı University, Material Science and Engineering Department, \\ Orhanl, Tuzla, Istanbul 34956 Turkey \\ ${ }^{3}$ SiOnyx Inc., 100 Cummings Center, Beverly, Massachusetts 01915 USA \\ ${ }^{4}$ Harvard University, Department of Physics and Division of Engineering and Applied \\ Sciences, 29 Oxford Street, Cambridge, Massachusetts 02138 USA \\ aserpenguzel@ku.edu.tr
}

\begin{abstract}
Room temperature visible and near-infrared photoluminescence from black silicon has been observed. The black silicon is manufactured by shining femtosecond laser pulses on silicon wafers in air, which were later annealed in vacuum. The photoluminescence is quenched above $120 \mathrm{~K}$ due to thermalization and competing nonradiative recombination of the carriers. The photoluminescence intensity at $10 \mathrm{~K}$ depends sublinearly on the excitation laser intensity confirming band tail recombination at the defect sites.
\end{abstract}

Keywords: band tail recombination, black silicon, laser spectroscopy, luminescence, optical communication, photoluminescence, quenching, recombination, silicon photonics.

\section{INTRODUCTION}

Silicon is the most widely available semiconducting material, since it is the second most abundant material on earth after oxygen. Silicon has been the material of choice for the microelectronics industry for more than half-a-century [1] since it is a relatively inexpensive, and well understood material for producing microelectronic devices [2]. Silicon based electrophotonic integrated circuit (EPIC), i.e., optoelectronic IC (OEIC) [3] is the natural evolution of the microelectronic IC with the added benefit of photonic capabilities.

Although silicon photonics is less well developed as compared to the direct bandgap III-V semiconductor photonics; silicon is poised to make a serious impact on the optical communications [4]. Silicon, with a near-infrared indirect bandgap of $1.1 \mathrm{eV}$ is transparent in the optical communication wavelengths greater than $1.1 \mu \mathrm{m}$, and is a suitable high refractive index optoelectronic group IV material. Therefore, silicon photonics [5] is experiencing a rapid growth, [6] which is in part driven by the need for low cost photonic devices [7] and the need for high speed intrachip communication [8,9]. Recent progress in silicon photonics is being heralded by the observation of first the Raman gain $[10,11]$ then stimulated Raman scattering (SRS) [12] in a crystalline silicon waveguide, SRS "lasing" first in pulse $[13,14]$ modulated [15] and later in CW [16] silicon Raman "lasers," and finally the hybrid silicon Raman "laser" [17]. Additionally, silicon modulators [18,19] have been developed first using a metal-oxide-semiconductor (MOS) capacitor [20], a Mach-Zehnder [21] configuration, SRS [22], and a microring [23] configuration. Recently, a silicon microring based wavelength converter has been realized [24]. Racetracks [25], microrings [26], waveguides with feedback [27, 28], and microspheres [29] are some of the resonator geometries pursued for silicon lightwave circuits (SLC's). With well established CMOS processing techniques, it will be possible to integrate lasers, waveguides, modulators, switches, wavelength converters, and photodetectors into silicon motherboards [30] for long haul, metro, local area, interchip, and even intrachip optical communication applications [31]. 
However, most of these discrete or integrated optoelectronic devices are fabricated using crystalline silicon. Black silicon [32] is a relatively novel material, which is obtained by femtosecond [33] or nanosecond [34] laser processing of crystalline silicon surfaces in the presence of a halogen containing gas such as $\mathrm{SF}_{6}$ or a variety of ambient gases such as $\mathrm{Cl}_{2}, \mathrm{~N}_{2}$, air [35], $\mathrm{H}_{2} \mathrm{~S}, \mathrm{H}_{2}$ [36], and $\mathrm{SiH}_{4}$ [37], or immersed in water [38], or coated with other group VI elements (chalcogens) such as selenium and tellurium [39]. In this process the flat, mirrorlike surface of a silicon wafer is transformed into a forest of microscopic spikes.

Black silicon has increased absorbance of approximately $90 \%$ from the near-ultraviolet to the near-infrared [40]. This absorbance resulted in high sensitivity infrared photodetectors [41], high quantum efficiency avalanche photodiodes (APDs) [42], and high sensitivity infrared photodiodes [43]. Additionally, black silicon has potential applications such as magnetizeable biodetectors [44], superhydrophobic surfaces [45], and microfluidic devices [46]. Regular arrays of black silicon has been produced for other potential device applications [47]. Black silicon also exhibits visible and near-infrared luminescence [48]. In this paper, we are reporting on the temperature and laser intensity dependence of the visible and nearinfrared photoluminescence from black silicon in order to fully characterize and optimize the material in the pursuit of obtaining novel nanophotonic devices.

\section{SAMPLE FABRICATION AND MORPHOLOGY}

Black silicon samples, which are used in our experiments, are manufactured by shining a series of very short, very intense laser pulses at a silicon surface in air. In the presence of the laser light, air reacts with the silicon surface and etches away some of it, leaving a pattern of conical spikes behind. The spiked surface is strongly light-absorbing: the surface of silicon, normally gray and shiny, turns deep black; hence the name black silicon.

Ordinarily, silicon absorbs a moderate amount of visible light, but a substantial amount of visible light is reflected as well, and infrared and ultraviolet light are transmitted through silicon or reflected from it with very little absorption. Spiked silicon surfaces, in contrast, absorb nearly all light at wavelengths ranging from the ultraviolet to the infrared.

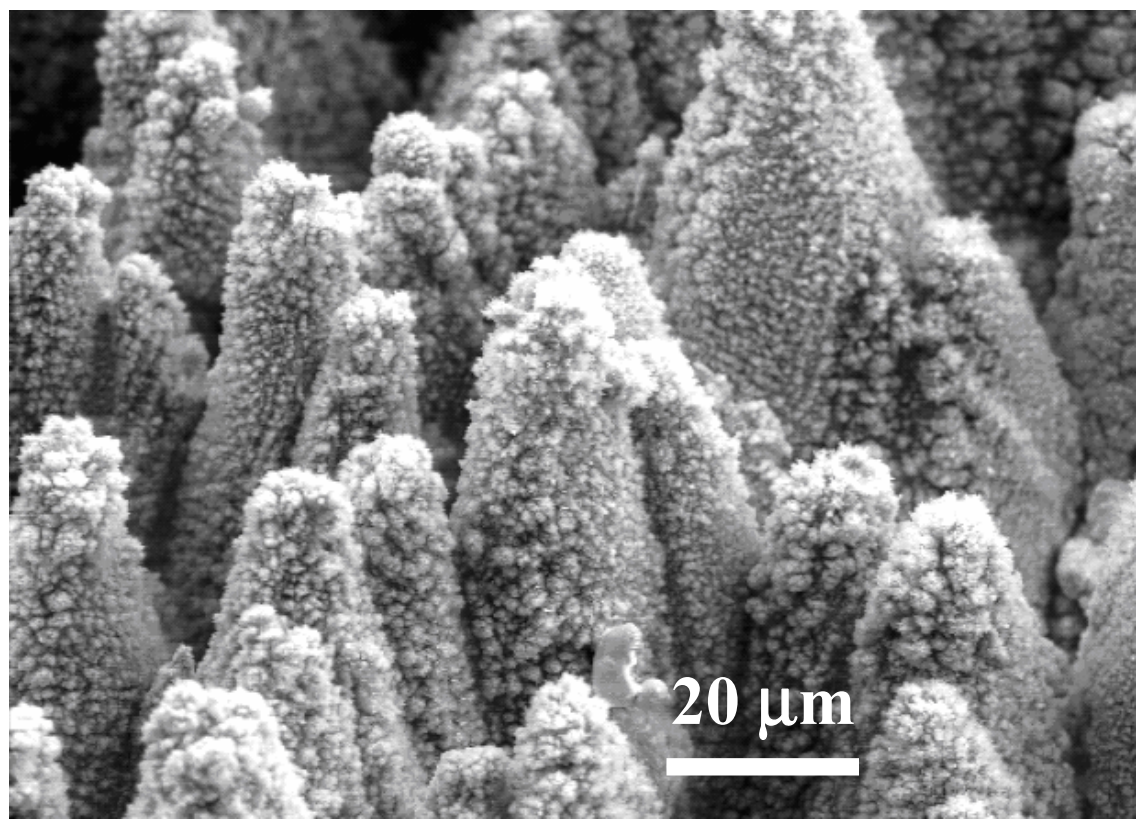

Fig. 1a. Low resolution SEM of the black silicon surface. 


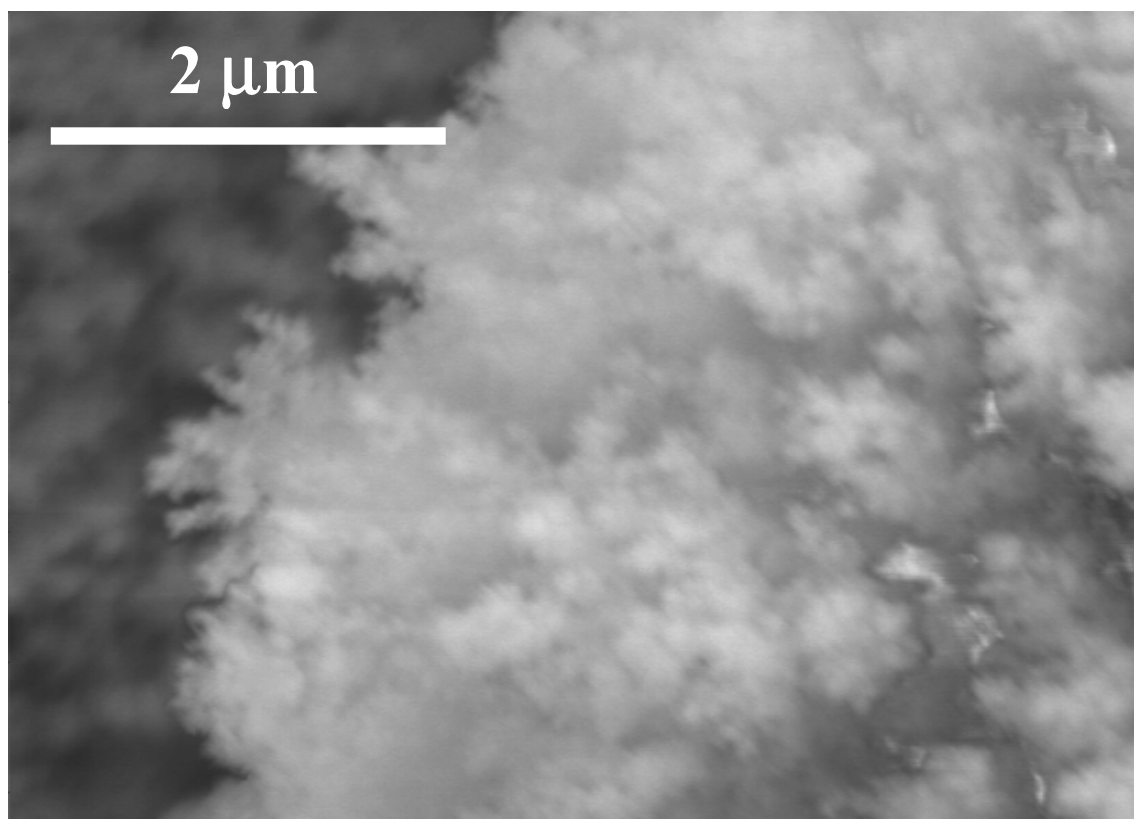

Fig. 1b. High resolution SEM of the black silicon surface.

In the femtosecond laser etching setup, the laser is aligned so that it is incident perpendicular to the (111) silicon wafer surface. An x-y translation stage was used to scan the (111) silicon wafer surface with the focused laser beam. The laser used was a Ti:sapphire pulse laser, with $100 \mathrm{fs}$ pulse duration, $800 \mathrm{~nm}$ wavelength, $1 \mathrm{kHz}$ repetition rate, and 22 $\mathrm{kJ} / \mathrm{cm}^{2}$ fluence. After laser microstructuring, the black silicon sample was annealed at $900^{\circ}$ for 3 hours in vacuum.

Scanning electron microscopy (SEM) has been performed on the black silicon samples to characterize the morphology of the surface and to analyze origin of the luminescence. Figure 1 shows SEM pictures of the black silicon surface. Tens of micrometers tall blunt conical microstructures are observed. These microstructures are covered with dendritic nanostructures roughly 10-100 nm in size, which disappear upon annealing. Room temperature visible and near infrared luminescence has previously been observed in these annealed samples [48].

\section{EXPERIMENTAL PHOTOLUMINESCENCE SETUP}

Figure 2 shows a schematic of the experimental photoluminescence (PL) setup. The black silicon sample is attached to copper holder, which is placed in a closed cycle cryostat system. The closed cycle cryostat system is used to control the sample temperature from $10 \mathrm{~K}$ to 300 $\mathrm{K}$. The pump laser is the second harmonic of a multimode Nd:YAG laser operating at a wavelength of $532 \mathrm{~nm}$ with a pulse duration of $10 \mathrm{~ns}$ and a repetition rate of $10 \mathrm{~Hz}$. The pump laser is incident on the sample at an angle of incidence of $17^{\circ}$. The PL signal is collected with a telescope through a holographic notch filter operating at $532 \mathrm{~nm}$. The collected PL signal is imaged onto an optical fiber bundle, which acts as the entrance slit of the spectrometer, whose output is fed to a sensitive GaAs photomultiplier tube (PMT). A lock-in-amplifier operating at $10 \mathrm{~Hz}$ provides the necessary electronic gain to the PMT output in phase with the laser pulse. The digital oscilloscope is used for monitoring and optimizing the PMT signal. All of the measurement and test devices are computer controlled and the data is acquired digitally. 


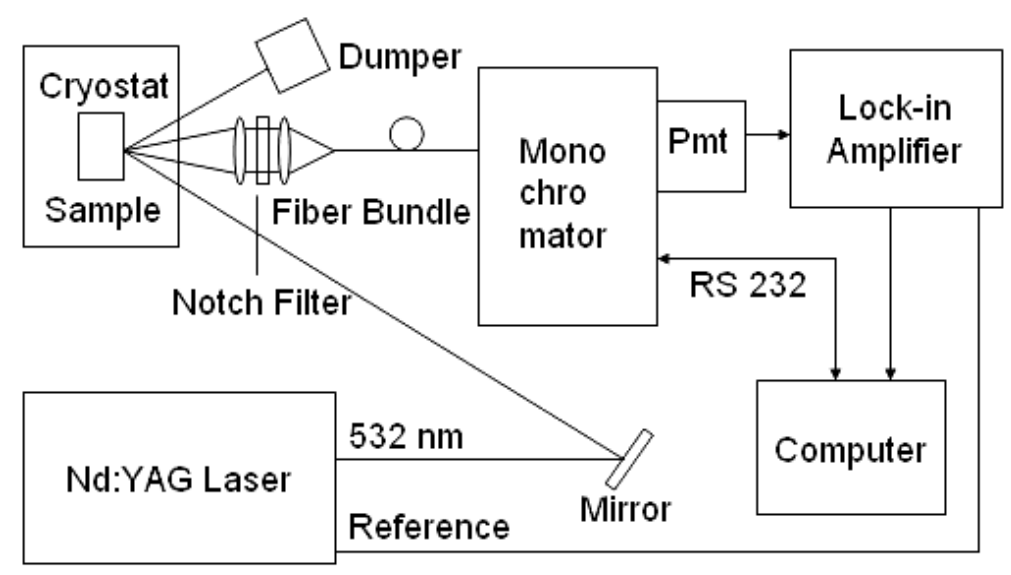

Fig. 2. The schematic of the experimental setup.

\section{TEMPERATURE DEPENDENCE OF THE LUMINESCENCE}

Figure 3 shows the PL spectra of the black silicon measured in the $550-850 \mathrm{~nm}$ wavelength and in the $10-300 \mathrm{~K}$ temperature range at a constant excitation laser intensity of $0.1 \mathrm{~W} \mathrm{~cm}^{-2}$. A broad PL band centered at $630 \mathrm{~nm}\left(E_{p}=1.968 \mathrm{eV}\right)$ at $10 \mathrm{~K}$ is observed. The PL spectra have approximately Gaussian lineshapes. The noise in the PL spectra is due to the intensity fluctuations of our multimode pulse Nd:YAG laser operating at $532 \mathrm{~nm}$. The PL intensity decreases with increasing temperature indicating that there is a quenching process through nonradiative recombination [49].

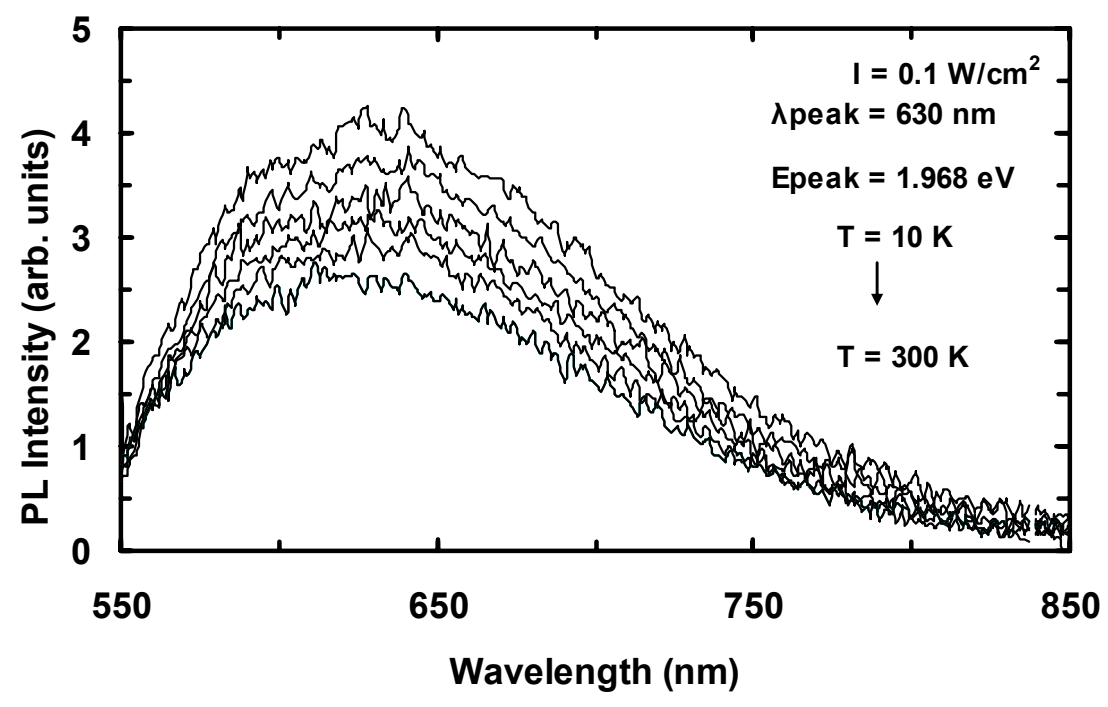

Fig. 3. PL spectra of black silicon in the $10-300 \mathrm{~K}$ range.

The variation of the PL intensity with respect to temperature is plotted in the Fig. 4. In the $10-120 \mathrm{~K}$ range, the PL intensity decreases slowly. Above $120 \mathrm{~K}$, however, the PL intensity decreases at a larger rate due to thermal quenching by nonradiative recombination processes 
[50]. The thermal quenching is associated with the mobility induced quenching of the band tail states. These band tail states of black silicon are associated with the presence of structural defects and impurities [51].

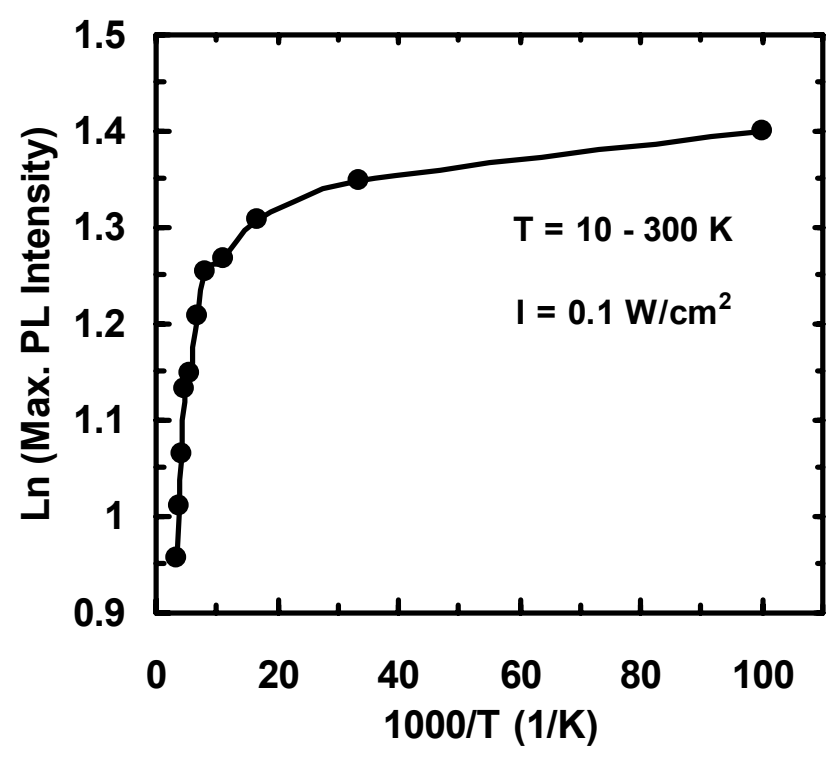

Fig. 4. Temperature dependence of black silicon PL intensity at the emission band maximum. Intensive quenching starts at $120 \mathrm{~K}$.

\section{INTENSITY DEPENDENCE OF THE LUMINESCENCE}

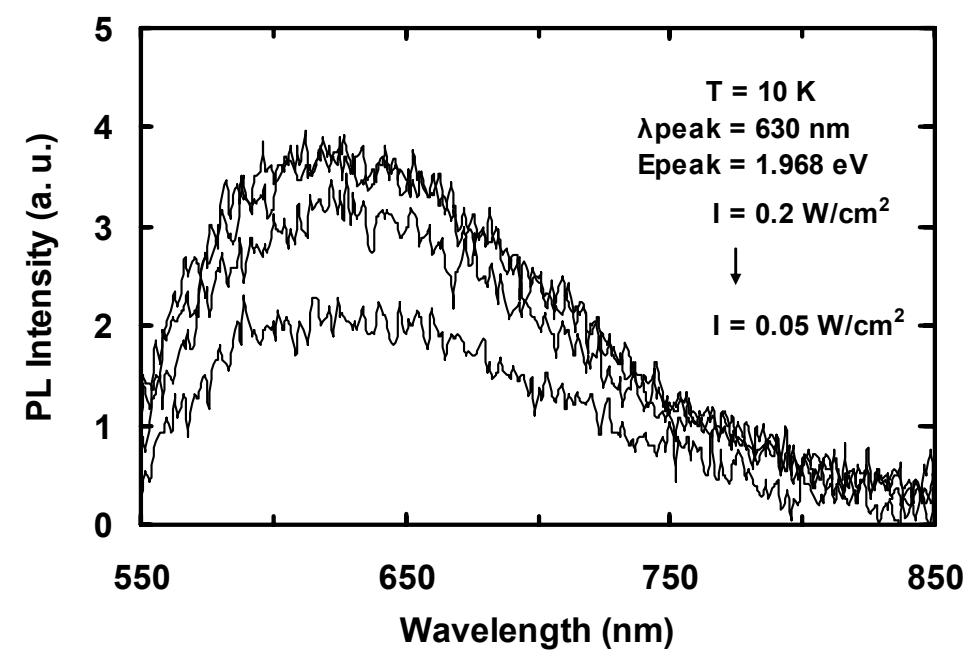

Fig. 5. PL spectra of black silicon at $0.05-0.2 \mathrm{~W} \mathrm{~cm}^{-2}$ laser intensities at $10 \mathrm{~K}$. 
Figure 5 shows the PL spectra for different laser intensities at $10 \mathrm{~K}$. As the laser intensity is increased the PL intensity increases. The intensity variation of the emission band versus the excitation laser intensity at $\mathrm{T}=10 \mathrm{~K}$ is investigated and plotted in the Fig. 6. The experimental data can be fitted by a simple power law, I $\sim \mathrm{L}^{\gamma}$, where I is the PL intensity, $\mathrm{L}$ the excitation laser intensity, and $\gamma$ a dimensionless exponent. It was found that, the PL intensity increases sublinearly (i.e., $\gamma=0.44$ ) with respect to the excitation laser intensity. For an excitation laser photon with an energy exceeding the band gap energy, the coefficient $\gamma$ is generally $1<\gamma<2$ for exciton emission; and $\gamma \leq 1$ for free to/from bound or bound to bound state recombination [52]. Thus, the obtained value of $\gamma=0.44$ at $10 \mathrm{~K}$ suggest that the PL in black silicon is due to bound to bound band tail recombination.

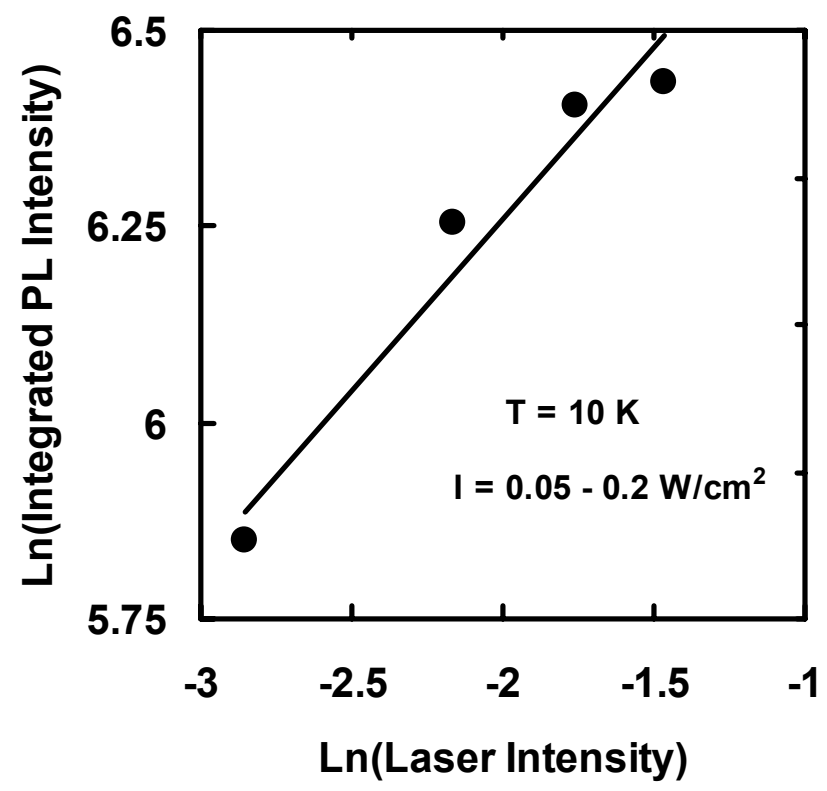

Fig. 6. Dependence of black silicon integrated PL intensity versus excitation laser intensity at $10 \mathrm{~K}$. The solid curve gives the theoretical fit using Eq. $\mathrm{I}=\mathrm{L}^{\gamma}$.

\section{CONCLUSIONS}

Photoluminescence (PL) properties of black silicon were studied at room temperature and at low temperatures. The PL intensity decreases with increasing temperature. Intensive quenching due to increased carrier mobility and nonradiative recombination starts at $120 \mathrm{~K}$, indicating that the PL is due to band tail recombinations in the defect states. Additionally, the sublinear variation of the PL intensity versus the excitation laser intensity at $10 \mathrm{~K}$ indicates that the PL is due to bound to bound state recombination at these band tail defect sites. The visible and near-infrared PL spectrum of the black silicon makes it a promising novel material for short haul optical communication applications.

\section{Acknowledgments}

We would like to acknowledge the partial support of this research by Koç University Harvard University Joint Research Program, and the European Commission FP6-IST 511616, Network of Excellence NanoPHOtonics to REalization of MOlecular Scale Technologies (PHOREMOST) and FP6-IST 003887, Network of Excellence on Micro Optics (NEMO). 


\section{References}

[1] L. Pavesi, "Will silicon be the photonic material of the next millennium?" J. Phys: Condens. Matter 15, 1169-1196 (2003) [doi:10.1088/0953-8984/15/26/201].

[2] C. Gunn, "CMOS photonics for high-speed interconnects," IEEE Micro 26, 58-66 (2006) [doi:10.1109/MM.2006.32].

[3] G. Guillot and L. Pavesi, Optical Interconnects: The Silicon Approach, Springer Verlag, Berlin (2006) [doi: 10.1007/978-3-540-28912-8].

[4] H. Zimmermann, Integrated Silicon Optoelectronics, Springer Verlag, Berlin (2000).

[5] G.T. Reed and A.P. Knights, Silicon Photonics: An Introduction, Wiley, New York (2004) [doi:10.1002/0470014180].

[6] R. Soref, "The past, present, and future of silicon photonics," IEEE J. Sel. Top. Quantum Electron. 12, 1678-1687 (2006) [doi:10.1109/JSTQE.2006.883151].

[7] M. Paniccia, M. Morse, and M. Salib, "Integrated Photonics," in Silicon Photonics, L. Pavesi and D. J. Lockwood, Eds., pp. 51-85, Springer Verlag, Berlin, 51-88 (2004) ) [doi: 10.1007/b11504].

[8] G. T. Reed, G. Z. Mashanovich, W. R. Headley, S. P. Chan, B. D. Timotijevic, and F. Y. Gardes, "Silicon photonics: are smaller devices always better?" Japanese J. Appl. Phys. 45, 6609-6615 (2006) [doi:10.1143/JJAP.45.6609].

[9] L. C. Kimerling, L. Dal Negro, S. Saini, Y. Yi, D. Ahn, S. Akiyama, D. Cannon, J. Liu, J.G. Sandland, D. Sparacin, J. Michel, K. Wada, and M.R. Watts, "Monolithic Silicon Microphotonics," in Silicon Photonics, L. Pavesi and D. J. Lockwood, Eds., pp. 89-119 Springer Verlag, Berlin, 89-119 (2004) [doi: 10.1007/b11504].

[10] A. Liu, H. Rong, M. Paniccia, O. Cohen, and D. Hak, "Net optical gain in a low loss silicon-on-insulator waveguide by stimulated Raman scattering," Opt. Exp. 12, 42614268 (2004) [doi:10.1364/OPEX.12.004261].

[11] H. Rong, A. Liu, R. Nicolaescu, and M. Paniccia, "Raman gain and nonlinear optical absorption measurements in a low-loss silicon waveguide," Appl. Phys. Lett. 85, 21962198 (2004) [doi:10.1063/1.1794862].

[12] R. Jones, H. Rong, A. Liu, A. Fang, and M. Paniccia, "Net continuous wave optical gain in a low loss silicon-on-insulator waveguide by stimulated Raman scattering," Opt. Exp.13, 519-525 (2005) [doi:10.1364/OPEX.13.000519].

[13] O. Boyraz and B. Jalali, "Demonstration of a silicon Raman laser," Opt. Exp.12, 52695273 (2004) [doi:10.1364/OPEX.12.005269].

[14] H. Rong, A. Liu, R. Jones, O. Cohen, D. Hak, R. Nicolaescu, A. Fang, and M. Paniccia, "An all-silicon Raman laser," Nature 433, 292-294 (2005) [doi:10.1038/nature03273].

[15] O. Boyraz and B. Jalali, "Demonstration of directly modulated silicon Raman laser," Opt. Exp.13, 796-800 (2005) [doi:10.1364/OPEX.13.000796].

[16] H. Rong, R. Jones, A. Liu, O. Cohen, D. Hak, A. Fang, and M. Paniccia, "A continuous-wave Raman silicon laser," Nature 433, 725-728 (2005) [doi:10.1038/nature03346].

[17] A. W. Fang, H. Park, O. Cohen, R. Jones, M. J. Paniccia, and J. E. Bowers, "Electrically pumped hybrid AlGaInAs-silicon evanescent laser," Opt. Exp. 14, 92039210 (2006) [doi:10.1364/OE.14.009203].

[18] A. Liu, D. Samara-Rubio, L. Liao, and M. Paniccia, "Scaling the modulation bandwidth and phase efficiency of a silicon optical modulator," IEEE J. Sel. Top. Quantum Electron. 11, 367-372 (2005) [doi:10.1109/JSTQE.2005.845618].

[19] L. Liao, A. Liu, R. Jones, D. Rubin, D. Samara-Rubio, O. Cohen, M. Salib, and M. Paniccia, "Phase modulation efficiency and transmission loss of silicon optical phase shifters," IEEE J. Quantum Electron. 41, 250-257 (2005) [doi:10.1109/JQE.2004.840079]. 
[20] A. Liu, R. Jones, L. Liao, D. Samara-Rubio, D. Rubin, O. Cohen, R. Nicolaescu, and M. Paniccia, "A high-speed silicon optical modulator based on a metal-oxidesemiconductor capacitor," Nature 427, 615-618 (2004) [doi:10.1038/nature02310].

[21] L. Liao, D. Samara-Rubio, M. Morse, A. Liu, D. Hodge, D. Rubin, U. D. Keil, and T. Franck, "High speed silicon Mach-Zehnder modulator," Opt. Exp.13, 3129-3135 (2005) [doi:10.1364/OPEX.13.003129].

[22] R. Jones, A. Liu, H. Rong, M. Paniccia, O. Cohen, and D. Hak, "Lossless optical modulation in a silicon waveguide using stimulated Raman scattering," Opt. Exp.13, 1716-1723 (2005) [doi:10.1364/OPEX.13.001716].

[23] Q. Xu, S. Manipatruni, B. Schmidt, J. Shakya, and M. Lipson, "12.5 Gbit/s carrierinjection-based silicon micro-ring silicon modulators," Opt. Exp. 15, 431-436 (2007).

[24] S. F. Preble, Q. Xu, and M. Lipson, "Changing the colour of light in a silicon resonator," Nature Photon. 1, 293-296 (2007) [doi:10.1038/nphoton.2007.72].

[25] B. D. Timotijevic, F. Y. Gardes, W. R. Headley, G. T. Reed, M. J. Paniccia, O. Cohen, D. Hak, and G. Z. Masanovic, "Multi-stage racetrack resonator filters in silicon-oninsulator," J. Optics A 8, S473-S476 (2006) [doi: 10.1088/1464-4258/8/7/S25].

[26] Q. F. Xu and M. Lipson, "All-optical logic based on silicon micro-ring resonators," Opt. Exp. 15, 924-929 (2007) [doi:10.1364/OE.15.000924].

[27] B. Jalali, "Teaching silicon new tricks," Nature Photon. 1, 193-195 (2007) [doi:10.1038/nphoton.2007.35].

[28] A. Alduino and M. Paniccia, "Interconnects - wiring electronics with light," Nature Photon. 1, 153-155 (2007) [doi:10.1038/nphoton.2007.17].

[29] Y. O. Yilmaz, A. Demir, A. Kurt, and A. Serpengüzel, "Optical channel dropping with a silicon microsphere," IEEE Photon. Technol. Lett. 17, 1662-1664 (2005) [doi:10.1109/LPT.2005.850896].

[30] B. Jalali, S. Yegnanarayanan, T. Yoon, T. Yoshimoto, I. Rendina, and F. Coppinger, "Advances in silicon on insulator optoelectronics," IEEE J. Select. Top. Quantum Electron. 4, 938-947 (1998) [doi:10.1109/2944.736081].

[31] B. J. Offrein, R. Germann, F. Horst, H. W. M. Salemink, R. Beyerl, and G. L. Bona, "Resonant coupler-based tuneable add-after-drop filter in silicon-oxynitride technology for WDM networks," IEEE J. Select. Top. Quantum Electron. 5, 1400-1406 (1999) [doi:10.1109/2944.806897].

[32] T.-H. Her, R. J. Finlay, C. Wu, S. Deliwala, and E. Mazur, "Microstructuring of silicon with femtosecond laser pulses," Appl. Phys. Lett. 73, 1673-1675 (1998) [doi:10.1063/1.122241].

[33] T.-H. Her, R.J. Finlay, C. Wu, and E. Mazur, "Femtosecond laser-induced formation of spikes on silicon," Appl. Phys. A 70, 383-385 (2000) [doi:10.1007/s003390051052].

[34] C. H. Crouch, J. E. Carey, J. M. Warrender, M. J. Aziz, E. Mazur and F. Y. Genin, "Comparison of structure and properties of femtosecond and nanosecond laserstructured silicon," Appl. Phys. Lett. 84, 1850-1852 (2004) [doi:10.1063/1.1667004].

[35] R. J. Younkin, J. E. Carey, E. Mazur, J. A. Levinson, and C.M. Friend, "Infrared absorption by conical silicon microstructures made in a variety of background gases using femtosecond-laser pulses," J. Appl. Phys. 93, 2626-2629 (2003) [doi:10.1063/1.1545159].

[36] B. R. Tull, J. E. Carey, M. A. Sheehy, C. M. Friend, and E. Mazur, Formation of silicon nanoparticles and web-like aggregates by femtosecond laser ablation in a background gas," Appl. Phys. A 83, 341-346 (2006) [doi: 10.1007/s00339-006-3502-7].

[37] M. A. Sheehy, L. Winston, J. E. Carey, C. M. Friend and E. Mazur, The role of the background gas in the morphology and optical properties of laser-microstructured silicon," Chem. Mater. 17, 3582-3586 (2005) [doi: 10.1021/cm049029i]. 
[38] M. Shen, C. H. Crouch, J. E. Carey, and E. Mazur, "Femtosecond laser-induced formation of submicrometer spikes on silicon in water," Appl. Phys. Lett. 85, 56945696 (2004) [doi: 10.1063/1.1828575].

[39] M. Sheehy, B. Tull, C. Friend, and E. Mazur, "Chalcogen doping of silicon via femtosecond laser irradiation," Mat. Sci. Eng. B 137, 289-294 (2006) [doi: 10.1016/j.mseb.2006.10.002].

[40] C. H. Crouch, J. E. Carey, M. Shen, E. Mazur, and F. Y. Genin, "Infrared absorption by sulfur-doped silicon formed by femtosecond laser irradiation," Appl. Phys. A 79, 16351641 (2004) [doi: 10.1007/s00339-004-2676-0].

[41] Z. Huang, J. E. Carey, M. Liu, X. Guo, E. Mazur, and J. C. Campbell, "Microstructured silicon photodetector," Appl. Phys. Lett. 89, 033506 (2006). [doi: 10.1063/1.2227629]

[42] R. A. Myers, R. Farrell, A. M. Karger, J. E. Carey, and E. Mazur, "Enhancing nearinfrared avalanche photodiode performance by femtosecond laser microstructuring," Appl. Opt. 45, 8825-8831 (2006) [doi:10.1364/AO.45.008825].

[43] J. E. Carey, C. H. Crouch, M. Shen, and E. Mazur, "Visible and near-infrared responsivity of femtosecond-laser microstructured silicon photodiodes," Opt. Lett. 30, 1773-1775 (2005).

[44] T. R. Polte, M. Shen, J. Karavitis, M. Montoya, J. Pendse, S. Xia, E. Mazur, and D. E. Ingber, "Nanostructured magnetizeable materials that switch cells between life and death," Biomaterials 28, 2783-2790 (2007) [doi:10.1016/j.biomaterials.2007.01.045].

[45] T. Baldacchini, J. E. Carey, M. Zhou, and E. Mazur, "Superhydrophobic surfaces prepared by microstructuring of silicon using a femtosecond laser," Langmuir 22, 4917-4919 (2006) [doi:10.1021/la053374k].

[46] B. R. Tull, J. E. Carey, E. Mazur, J. P. McDonald, and S. M. Yalisove, "Surface morphologies of silicon surfaces after femtosecond laser irradiation," Mat. Res. Soc. Bull. 31, 626-633 (2006).

[47] M. Shen, C. H. Crouch, J. E. Carey, R. J. Younkin, E. Mazur, M. A. Sheehy, and C. M. Friend, "Formation of regular arrays of silicon microspikes by femtosecond laser irradiation through a mask," Appl. Phys. Lett. 82, 1715-1717 (2003) [doi: $10.1063 / 1.1561162]$.

[48] C. Wu, C. H. Crouch, L. Zhao, and E. Mazur, "Visible luminescence from silicon surfaces microstructured in air," Appl. Phys. Lett. 81, 1999-2001 (2002) [doi:10.1063/1.1504868].

[49] S. Tong, X. N. Liu, T. Gao, X. M. Baa, Y. Chang, W. Z. Shen, and W. G. Tang, "Temperature dependence of visible photoluminescence from PECVD nanocrystallites embedded in amorphous silicon films," Solid State Commun. 104, 603-607 (1997) [doi: 10.1016/S0038-1098(97)00391-8].

[50] R. A. Street, Hydrogenated Amorphous Silicon, Cambridge University Press, Cambridge, (1991).

[51] C. Wu, C. H. Crouch, L. Zhao, J. E. Carey, R. J. Younkin, J. A. Levinson, E. Mazur, R. M. Farrel, P. Gothoskar, and A. Karger, "Near-unity below-band gap absorption by microstructured silicon," Appl. Phys. Lett. 78, 1850-1852 (2001) [doi: 10.1063/1.1358846].

[52] T. Schmidt, K. Lischka, and W. Zulehner, "Excitation-power dependence of the nearband-edge photoluminescence of semiconductors," Phys. Rev. B 45, 8989-8994 (1992) [doi:10.1103/PhysRevB.45.8989]. 